$>$ L'intérêt des approches économiques dans le domaine de la santé réside dans la possibilité de hiérarchiser, sur la base de deux critères (efficacité médicale et coûts), les programmes médicaux susceptibles d'être menés, et d'aider à fixer des objectifs de santé publique, à moyen ou à long terme, compatibles avec les ressources disponibles. Cet article présente une revue de la littérature spécifique des principaux travaux d'évaluation économique qui se sont efforcés de contribuer à la définition de politiques optimales en matière de dépistage et de traitement de l'hépatite $C$. Les résultats de ces évaluations économiques montrent d'une part, que, malgré un coût économique immédiat important, le dépistage de certaines populations à risque est source à long terme d'économies par rapport à l'absence de dépistage, et suggèrent, d'autre part, que les traitements, y compris les plus coûteux et performants, aboutissent à sauver des années de vie pour des rapports qui ne sont jamais supérieurs à ceux généralement admis comme limite pour les interventions dans le domaine de la santé. <

\title{
Évaluation économique du dépistage et du traitement de I'hépatite C
}

\section{Sandrine Loubière, Michel Rotily, Jean-Paul Moatti}

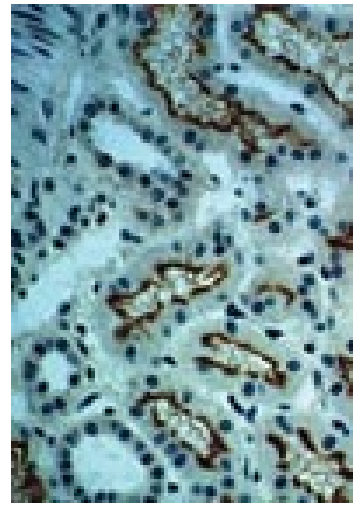

$650000[1,2]$, parmi lesquelles seule une minorité aurait connaissance de son statut sérologique [3]. Seuls $20 \%$ des patients infectés par le VHC vont guérir spontanément, les $80 \%$ restants évoluant vers un portage chronique du virus, puis éventuellement vers une cirrhose et un cancer hépatocellulaire. Des études récentes laissent entrevoir une augmentation constante de l'incidence de la mortalité par hépatocarcinome jusqu'en 2020 en l'absence de traitement. En 1979, on dénombrait 450 décès pour cause de cancer du foie. $\varepsilon n$ 1985, 1450 décès étaient associés aux cancers du foie, et 2100 décès sont prévus en 2001 [4].

Cependant, les traitements actuels, notamment I'association interféron-ribavirine et plus récemment le recours à I'interféron pégylé (IFN-PEG), laissent envisager une réduction significative de la morbidité et de la mortalité dues à I'hépatite $C$, à des coûts toutefois très élevés. Néanmoins, le nombre de personnes annuellement mises sous traitement (de l'ordre de 3500 à 5000 patients) reste limité en regard de l'étendue de l'épidémie. 
Plusieurs arguments peuvent être avancés pour expliquer ce décalage : une proportion de patients ne relèvent effectivement pas d'un traitement, soit du fait de la forme clinique, soit du fait de contre-indications aux traitements ; les effets secondaires du traitement pour le VHC peuvent représenter un frein à l'acceptation de ce dernier; une autre raison pourrait tenir au fait que seule une minorité des personnes infectées effectuerait spontanément une démarche de dépistage et aurait connaissance de son statut sérologique [3].

Aussi, étant donnés les enjeux en terme de morbidité et de mortalité, et le coût social de l'infection par le VHC, le recours à l'évaluation économique de cette infection apparaît pleinement justifié [5]. En effet, il faut rappeler que toute décision clinique d'engager un traitement ou une investigation diagnostique revient implicitement à sacrifier la possibilité de consacrer les ressources ainsi consommées à d'autres usages, c'est-à-dire à satisfaire d'autres besoins ou les besoins d'autres individus.
Aussi, les questions qui se posent aujourd'hui en terme de santé publique sont : qui dépister pour le VHC? Qui traiter? À quel coût?

Dans cet article, nous présentons une revue de la littérature des principaux travaux d'évaluation économique qui se sont efforcés de contribuer à la définition de politiques optimales en matière de dépistage et de traitement de l'hépatite $C$.

\section{L'évaluation du dépistage et la loi des rendements décroissants}

En 1994, les premiers écrits apparaissent sur l'évaluation économique de l'infection par le VHC. Ils vont s'intéresser distinctement à deux champs d'application : d'un côté, le dépistage du VHC et, de l'autre, le traitement de cette infection.

En 1991, le dépistage du VHC devient systématique chez les donneurs de sang. Les premiers travaux ont logique-

\begin{tabular}{|c|c|c|c|c|c|}
\hline Auteurs & Année & Objectifs de l'étude & Critères d'efficacité & $\begin{array}{l}\text { Critères du rapport } \\
\text { coût-efficacité }\end{array}$ & $\begin{array}{c}\text { Rapport } \\
\text { coût-efficacité } \\
€\end{array}$ \\
\hline Vergnon [6] & 1996 & $\begin{array}{l}\text { - ALAT et recherche anticorps anti-VHB } \\
\text { - ALAT et recherche anticorps anti-VHB } \\
\text { et VHC sur dons du sang }\end{array}$ & Donneurs positifs dépistés & Coût moyen & $\begin{array}{l}\cdot 2908 \\
\cdot 2680\end{array}$ \\
\hline Sailly [7] & 1997 & $\begin{array}{l}\text { - ELISA } 2^{e} \text { génération suivi d'un test } \\
\text { de confirmation en cas de résultat positif }\end{array}$ & $\begin{array}{l}\text { Donneurs vrais } \\
\text { positifs dépistés }\end{array}$ & Coût moyen & $\cdot 14620$ \\
\hline Laplane [9] & 1998 & $\begin{array}{l}\text { Stratégies fonction de : } \\
\text { - Prévalence }>7 \% \\
\text { - Présence de facteurs de risque } \\
\text { - Présence ALAT élevées }\end{array}$ & Individus dépistés & Coût moyen & $\begin{array}{l}\cdot 377 \\
\cdot 493 \\
\cdot 1107\end{array}$ \\
\hline Kaur $[10]$ & 1996 & - $\varepsilon L I S A+$ test immunoblot + ALAT & Individus dépistés & Coût moyen & $\cdot 1454$ \\
\hline Rotily [8] & 1997 & $\begin{array}{l}\text { Différentes stratégies combinant } \varepsilon \text { LISA, RIBA } \\
\text { et PCR en fonction de la prévalence } \\
\text { - } 70 \% \\
\text { - } 20 \% \\
\cdot 7 \% \\
\cdot 1,2 \%\end{array}$ & Individus vrais positifs & Coût moyen & $\begin{array}{l}\cdot 120 \\
\cdot 499 \\
\cdot 1295 \\
\cdot 7965\end{array}$ \\
\hline Loubière [14] & 2001 & $\begin{array}{l}\text { - } E L I S A \\
\text { - ELISA+PCR sur pools } \\
\text { - ELISA+PCR sur chaque don de sang }\end{array}$ & Années de vie gagnées & $\begin{array}{l}\text { Coût marginal } \\
\text { - Référence : ELISA } \\
\text { - Référence : ELISA }\end{array}$ & $\begin{array}{l}.85 \text { millions } \\
.891 \text { millions }\end{array}$ \\
\hline Loubière [27] & 1999 & $\begin{array}{l}\text { Différentes stratégies combinant } \varepsilon \text { LISA, RIBA } \\
\text { et PCR en fonction de la prévalence, dont : } \\
\text { - } \varepsilon L I S A \text {, puis deuxième } \varepsilon L I S A \\
\text { - Un seul ELISA }\end{array}$ & $\begin{array}{l}\text { Pathologies hépatiques } \\
\text { sévères évitées }\end{array}$ & $\begin{array}{l}\text { Coût marginal } \\
\text { - Référence : abstention } \\
\text { - Référence : ELISA, ELISA }\end{array}$ & $\begin{array}{l}\cdot 21700 \text { à } 46900 \\
\cdot 18430 \text { à } 99150\end{array}$ \\
\hline
\end{tabular}

Tableau I. Exemples d'évaluations économiques du dépistage du VHC. Ce tableau présente les principaux résultats des différentes études. Toutes les valeurs données ont été actualisées en coûts de I'an 2000. Lorsqu'il s'agit d'un coût marginal, la stratégie de référence est indiquée. ELISA : enzyme linked immunosorbent assay; RIBA : radio-immunobiologic assay. 


\begin{tabular}{|c|c|c|c|c|c|}
\hline Auteurs & Année & Objectifs de l'étude & Critères d'efficacité & $\begin{array}{l}\text { Critères du rapport } \\
\text { coût-efficacité }\end{array}$ & $\begin{array}{l}\text { Rapport } \\
\text { it-efficacité } \\
\quad €\end{array}$ \\
\hline Joliot [19] & 1996 & $\begin{array}{l}\text { - Absence de traitement } \\
\text { - IFN } 6 \text { mois } \\
\text { - IFN } 12 \text { mois } \\
\text { - IFN } 12 \text { mois et arrêt à } 3 \text { mois si non réponse }\end{array}$ & Cirrhoses évitées & $\begin{array}{l}\text { Coût marginal } \\
\text { - Référence : abstention } \\
\text { - Référence : IFN 6mois } \\
\text { - Référence : IFN 6mois }\end{array}$ & $\begin{array}{l}-40820^{1} \\
22510 \\
-35600\end{array}$ \\
\hline Michel [20] & 1996 & $\begin{array}{l}\text { Estimation du risque de cirrhose à } 8 \text { ans } \\
\text { pour trois stratégies de traitement : } \\
\text { - Abstention } \\
\text { - Traitement des hépatites chroniques actives } \\
\text { - Traitement de toutes les formes } \\
\text { d'hépatites chroniques }\end{array}$ & Cirrhoses évitées & $\begin{array}{l}\text { Coût marginal } \\
\text { - Référence : abstention } \\
\text { - Référence : traitement } \\
\text { des hépatites chroniques } \\
\text { actives }\end{array}$ & 45850 \\
\hline Bennett [21] & 1997 & $\begin{array}{l}\text { Traitement IFN } 6 \text { mois chez patients } \\
\text { atteints d'une hépatite chronique modérée }\end{array}$ & $\begin{array}{l}\text { Années de vie gagnées, } \\
\text { en fonction de l'âge au début } \\
\text { du traitement: } \\
\text { - } 20 \text { ans } \\
\text { - } 35 \text { ans } \\
\text { - } 70 \text { ans }\end{array}$ & $\begin{array}{l}\text { Coût marginal } \\
\text { - Référence : abstention } \\
\text { - Référence : abstention } \\
\text { - Référence : abstention }\end{array}$ & $\begin{array}{l} \\
.530 \\
\cdot 2170 \\
.70820\end{array}$ \\
\hline Shiell [22] & 1994 & $\begin{array}{l}\text { - Abstention } \\
\text { - Traitement des patients atteints } \\
\text { d'une hépatite chronique modérée } \\
\text { ou atteints d'une cirrhose }\end{array}$ & Années de vie gagnées & $\begin{array}{l}\text { Coût marginal } \\
\text { - Référence : abstention }\end{array}$ & .67300 \\
\hline Dusheiko [23] & 1995 & $\begin{array}{l}\text { - Abstention } \\
\text { - Traitement des patients atteints } \\
\text { d'une hépatite chronique modérée }\end{array}$ & Années de vie gagnées & $\begin{array}{l}\text { Coût marginal } \\
\text { - Référence : abstention }\end{array}$ & $\begin{array}{l}\cdot 4200 \text { à } \\
10300\end{array}$ \\
\hline $\operatorname{Kim}[24]$ & 1997 & $\begin{array}{l}\text { - Traitement IFN } 12 \text { mois } \\
\text { - Traitement IFN } 6 \text { mois }\end{array}$ & $\begin{array}{l}\text { PALY } \\
\text { (voir glossaire) }\end{array}$ & $\begin{array}{l}\text { Coût marginal } \\
\text { - Référence : abstention } \\
\text { - Référence : abstention }\end{array}$ & $\begin{array}{l}. \\
. \\
.\end{array} 25250$ \\
\hline Wong [25] & 1998 & $\begin{array}{l}\text { Stratégie de prise en charge en fonction } \\
\text { d'examens préalables dont: } \\
\text { - Biopsie } \\
\text { - Génotype } \\
\text { - PCR (seuil ARN VHC } \leq 32 \times 10^{5} \text { ) } \\
\text { - Aucun examen (traitement de toute infection) }\end{array}$ & QALY & $\begin{array}{l}\text { Coût marginal } \\
\text { - Dominée } \\
\text { - Dominée } \\
\text { - Référence : } \\
\text { PCR }\left(\text { ARN } \leq 3,5 \times 10^{5}\right) \\
\text { - Référence : } \\
\text { PCR }\left(\text { ARN } \leq 3,5 \times 10^{5}\right)\end{array}$ & $\begin{array}{r}.4870 \\
.14200\end{array}$ \\
\hline Wong [26] & 2000 & $\begin{array}{l}\text { Comparaison de : } \\
\text { - Traitement IFN } 12 \text { mois } \\
\text { - Traitement IFN + ribavirine } 6 \text { mois } \\
\text { - Traitement IFN + ribavirine } 12 \text { mois }\end{array}$ & Années de vie gagnées & $\begin{array}{l}\text { Coût marginal } \\
\text { - Référence : abstention } \\
\text { - Référence : abstention } \\
\text { - Référence : IFN + RIBA } 6 \text { mois }\end{array}$ & $\begin{array}{r}\cdot 2150 \\
\cdot 2355 \\
\cdot 22560\end{array}$ \\
\hline
\end{tabular}

Tableau II. Exemples d'évaluations économiques du traitement du VHC. Ce tableau présente les principaux résultats des différentes études. Toutes les valeurs données ont été actualisées en coûts de l'an 2000. Lorsqu'il s'agit d'un coût marginal, la stratégie de référence est indiquée. 
ment porté sur le coût de cet investissement pour les établissements de transfusion sanguine $[6,7]$ (Tableau 1). Vergnon et al. [6] ont évalué le dépistage des dons de sang reposant sur le dosage des ALAT (alanine aminotransférase) et la recherche d'anticorps antiVHB (virus de I'hépatite B) et anti-VHC, et ont estimé le coût moyen (voir glossaire) d'un tel dépistage de l'ordre de $2960 €\left(17600 \mathrm{MF}_{2000}\right)^{*}$, le coût global étant estimé à 8,55 millions d'Euro $\left(56 \mathrm{MF}_{2000}\right)$. Sailly et al. [7] ont étudié l'efficience d'un dépistage systématique des virus VIH, VHB, VHC et HTLV chez les donneurs de sang par un test ELISA suivi d'un test de confirmation en cas de résultat positif. Le coût global annuel engendré par la mise en œuvre d'une telle sécurité virologique était estimé à environ 51 millions d'Euros (335 MF) pour les quatre virus étudiés, et les coûts moyens par séroconversion évitée grâce au dépistage des dons à $14620 €$ (96000 F) pour le VHC, soit plus du double que pour le VHB (6555€, $43000 \mathrm{~F}$ ) mais beaucoup moins que pour le VIH (138000€, $905000 \mathrm{~F}$ ) et le HTVL (human T cell leukemia virus) (>1, 22 millions d'€- 8 MF.).

Avec le développement de l'épidémie à VHC, s'est posée la question de l'élargissement du dépistage à d'autres groupes de populations dits à risque, en fonction de la prévalence du VHC, des modalités optimales de ce dépistage, ainsi que de l'éventuel bien-fondé de son extension à la population générale [8-10]. Dans ce contexte précis du dépistage où les stratégies peuvent associer plusieurs tests diagnostiques en parallèle ou en série, avec des sensibilités et des spécificités propres, et également s'appliquer à différentes populations caractérisées par une certaine valeur de la prévalence du virus recherché, le décideur se trouve confronté à un phénomène récurrent en matière de santé : la loi des rendements décroissants (voir glossaire) [11, 12]. Dans une étude réalisée à l'occasion de la Conférence de Consensus sur la prévention et la prise en charge du VHC tenue en janvier 1997 à Paris [8], nous avons comparé les rapports coût/efficacité de différentes stratégies de dépistage correspondant aux diverses combinaisons envisageables des tests diagnostiques disponibles (Tableau 1). L'analyse montrait dans un premier temps que, quelle que soit la prévalence dans la population dépistée, les stratégies commençant par le dosage des ALAT en première intention se révélaient toujours dominées (efficacité plus faible pour un coût plus élevé) ; dans un second temps, la stratégie reposant sur un ELISA seul avait le meilleur rapport coût/efficacité, et ce quelle que soit la prévalence ; le coût par cas vrai posi-

* Afin de permettre des comparaisons entre les différentes études, toutes les valeurs données dans le texte ont été actualisées en coûts de l'an 2000. tif dépisté passant d'environ $1295 €$ (8 500 F) dans la population des transfusés (avec une prévalence estimée de l'ordre de $7 \%$ ) à $7965 €(52300 \mathrm{~F})$ en population générale (pour une prévalence de porteurs de l'ordre de $1,2 \%$ ). L'analyse soulignait cependant qu'à partir d'un niveau de prévalence supérieur à $8 \%$, correspondant à peu près à celui observé chez les transfusés et donc très largement dépassé chez les usagers de drogue par voie intraveineuse, des stratégies de dépistage utilisant la PCR (polymerase chain reaction) en première intention impliquaient une détérioration du rapport coût-efficacité qui pouvait apparaître «raisonnable», avec une importante réduction des faux positifs et des faux négatifs.

Ainsi, plus le dépistage est étendu à des groupes de population où la prévalence de l'affection recherchée est faible, ou bien plus la stratégie de dépistage multiplie les tests afin de réduire le « risque résiduel » d'erreurs (faux négatifs notamment) dans une même population, plus il faudra consacrer de ressources pour identifier un cas supplémentaire.

La révélation des rendements décroissants en matière de dépistage s'exprime au travers de la distinction entre coût moyen et coût marginal (voir glossaire) [5, 13]. Cette dernière estimation est essentielle dans la mesure où ce que I'on cherche à évaluer, lorsque l'on compare des stratégies entre elles, c'est la mesure des écarts entre l'efficacité, c'est-à-dire l'amélioration de la santé au niveau de la population visée, et le coût supplémentaire nécessaire pour produire cette amélioration de l'état de santé. La différence entre coût moyen et coût marginal a donc toute son importance lorsque I'on cherche à déterminer la stratégie qui permettra d'optimiser un résultat médical sous contrainte de ressources données.

Une illustration d'un cas « limite » de rendement décroissant a été fournie par la décision récente (octobre 2000) des pouvoirs publics français d'adjoindre systématiquement une recherche de I'ARN du VHC, par la technique de PCR, au test sérologique ELISA de troisième génération pour le dépistage du VHC dans les dons de sang. Une analyse coût-efficacité, réalisée préalablement dans le cadre d'un groupe d'experts mis en place par la Direction Générale de la Santé [14], avait montré que le coût additionnel (coût marginal) de la réduction du risque de contamination transfusionnelle permise par l'introduction de la PCR s'élèverait à 85 millions d'€ (560 millions de francs) par année de vie potentiellement gagnée si la PCR s'appliquait à des pools de 50 dons et à près de dix fois plus si le dépistage par PCR était effectué sur chaque don. Ces chiffres sont évidemment sans commune mesure avec les rapports 
coût/efficacité de l'ordre de $7600 €(50000$ F) par année de vie gagnée qui sont considérés aujourd'hui comme un plafond maximal en matière de sécurité routière ou qui correspondent aux avancées les plus récentes de l'hormonothérapie et de la chimiothérapie pour le cancer du sein chez les femmes pré-ménopausées [15].

Si la finalité d'un dépistage de masse - c'est-à-dire d'un test de dépistage proposé à une population apparemment en bonne santé - est d'améliorer les indicateurs épidémiologiques (mortalité et morbidité) des pathologies concernées en terme de santé publique, en distinguant les personnes probablement atteintes de celles probablement indemnes, il ne donne pas la garantie d'un apport positif et direct à la santé de tous les individus auxquels il est proposé. En ce sens, l'élargissement d'un programme de dépistage à d'autres populations à plus ou moins haut risque soulève généralement des problèmes d'équité $[16,17]$. Ainsi, dans certains cas, le dépistage peut présenter un bilan risque/bénéfice qui se révèle a posteriori défavorable pour certains individus, par exemple lorsque des faux positifs du dépistage du VHC induisent des actes invasifs potentiellement iatrogènes et une anxiété inutile chez des sujets en fait non atteints. C'est pourquoi il est indispensable, avant d'engager un programme de dépistage de masse, de s'assurer que les conséquences bénéfiques sont réelles et supérieures aux possibles effets pervers. L'analyse économique vise à contribuer à une telle « étude d'impact » du dépistage.

La mesure du bénéfice du dépistage supposerait de prendre en compte, non seulement son impact direct sur les individus qui peuvent bénéficier d'un traitement précoce, mais ses avantages en termes de réduction des « externalités » négatives liées au caractère transmissible du VHC, c'est-à-dire l'éventuelle limitation des comportements à risque de transmission du virus à autrui qui peut être induite par la connaissance par les sujets infectés de leur statut sérologique. Si la prise en compte des externalités du dépistage, qui implique de coupler l'évaluation économique avec des modélisations sophistiquées de la dynamique épidémiologique de transmission d'un virus, a pu être réalisée dans le cas de la vaccination contre l'hépatite $B$ [18], aucune évaluation économique en matière de VHC n'a été en mesure de couvrir cet aspect : il est vrai que le problème est rendu plus délicat par la difficulté d'estimer les modifications effectives de comportement que peut impliquer la prise de conscience d'un statut sérologique positif pour le VHC dans différents groupes exposés.
En tout état de cause, si les analyses coût-efficacité appliquées au dépistage du VHC ont déjà contribué à clarifier la question du « comment » (celle des modalités les plus efficientes en termes d'allocation des ressources une fois prise la décision de dépister dans un groupe donné), elles ne peuvent répondre à la question du « jusqu'où », c'est-à-dire du bien-fondé ou non de décider d'une politique de dépistage systématique, qu'en reliant l'évaluation économique du dépistage à celle des traitements, et donc de leur impact potentiel commun en termes de modifications de l'histoire naturelle de la maladie, et de la morbidité et de la mortalité qui lui sont associées.

\section{La prise en compte des conséquences à long terme du dépistage et la question du « jusqu'où ? »}

Comme nous l'avons montré, la plupart des analyses socio-économiques qui ont évalué le dépistage de l'hépatite $C$, l'ont fait de façon indépendante des conséquences à long terme de cette pratique, par exemple I'existence d'un traitement efficace. C'est au milieu des années 1990, avec l'apparition de nouveaux schémas thérapeutiques, que la littérature scientifique s'est intéressée plus particulièrement à l'évaluation économique d'alternatives thérapeutiques du VHC (Tableau II) [19-25]. En comparant trois stratégies de prise en charge (interféron $\alpha$ pendant soit 6 mois, soit 12 mois, soit 12 mois avec interruption à 3 mois en cas de non-réponse) à l'absence de traitement, Joliot et al. [19] ont établi la supériorité du traitement par interféron $\alpha$ par rapport à l'absence de traitement puisque celui-ci permet une économie nette dès lors qu'on prend en compte les coûts de prise en charge des cirrhoses qui ne seraient pas «évitées » en l'absence de traitement. Michel et al. [20] ont montré que le coût moyen par cas de cirrhose évité faisait plus que doubler dès lors qu'on traitait systématiquement toutes les formes d'hépatites plutôt que les seules formes actives (respectivement $90250 €$ versus $45887 €-592000$ F versus 301000 F), ce qui correspond à un coût marginal considérable de la première stratégie par rapport à la seconde de l'ordre de $780000 €$ ( 5 millions de Francs) par cirrhose supplémentaire évitée. L'intérêt du traitement en fonction de I'âge du patient au moment de la mise sous traitement a également été évalué [21]. Pour un patient âgé d'une vingtaine d'années, le coût par année de vie gagnée était estimé à $530 €(3470 \mathrm{~F})$, ce coût était multiplié par douze (70 $820 €$, soit 465000 Francs) dès lors que le patient était âgé de plus de 70 ans. En ce sens, les auteurs des 3 études précédemment citées se sont 
efforcés de répondre à la question du choix de la « taille » de l'intervention médicale, c'est-à-dire de seuils jusqu'où il est légitime d'allouer des ressources à l'extension d'une stratégie médicale donnée.

On peut également souligner que les estimations du coût par année de vie gagnée avec le traitement par interféron $\alpha$ diffèrent nettement, de $67300 €$ (442 000F) [22] à des valeurs environ dix fois moindres (10 300 et $4200 €$, soit $67600 \mathrm{~F}$ et 27500 F) [23], selon les différentes hypothèses émises sur le taux de progression de l'hépatite chronique vers la cirrhose et le taux de mortalité. Cette différence s'explique pour l'essentiel par la prise en compte, dans le deuxième cas, des coûts indirects liés à la morbidité qui augmentent d'autant les économies permises par le traitement en comparaison de son absence.

Kim et al. [24] ont comparé deux durées de traitement par interféron $\alpha$ à l'absence de traitement. Le schéma court ( 6 mois) permettait en moyenne un gain additionnel de 0,25 année ajustée sur la qualité de vie ( $(A L Y$ - voir glossaire) et le régime long (12 mois) de 0,37 QALY, correspondant respectivement à des coûts additionnels par PALY supplémentaire gagné de $4720 €$ (31000 F) et $6250 €(41000$ F). La comparaison de différentes modalités de décision de la mise en œuvre du traitement par interféron [25] a permis de montrer que la stratégie reposant sur la réalisation d'une PCR présentait le meilleur rapport coût-efficacité marginal (4 $870 €$ soit $32000 \mathrm{~F} /$ QALY), mais conduisait à ne pas traiter $36 \%$ des répondeurs potentiels à long terme. Par comparaison, la mise sous traitement systématique de tous les porteurs d'une hépatique chronique $\mathrm{C}$ impliquait une détérioration significative du ratio coût/efficacité (14 200 € soit 93000 F/QALY). La stratégie reposant sur une biopsie initiale était beaucoup plus coûteuse (>41 $900 €$ soit 275000 F/QALY), ne laissait échapper que $6 \%$ de répondeurs potentiels à long terme, mais augmentait le nombre de décès lié au caractère potentiellement iatrogène de cette procédure diagnostique (18 décès pour 100000 biopsies).

Les résultats des évaluations du traitement de l'infection par le VHC convergent pour suggérer que la prise en charge thérapeutique de cette infection est globalement coût-efficace, comparée d'une part à l'absence de traitement, d'autre part à d'autres stratégies couramment pratiquées pour d'autres pathologies, même en considérant un traitement empirique par interféron seul. L'évaluation économique de stratégies de traitement associant la ribavirine à l'interféron alpha recombinant ou de nouvelles formes d'interféron, et de stratégies de sélection de patients plus susceptibles de répondre aux traitements, permettra d'apporter des éléments décisionnels complémentaires. La première étude coût-efficacité comparant mono-thérapie et bithérapie montrait que le traitement par interféron et ribavirine, quoique a priori trois fois plus coûteux, permet une amélioration significative de l'efficacité thérapeutique pour une détérioration limitée du rapport coût/efficacité par rapport au traitement de 12 mois par interféron seul : le coût par année de vie gagnée supplémentaire grâce au passage de la mono- à la bithérapie est en effet estimé dans cette étude autour de $2560 €(16800 \mathrm{~F})$ [26].

Cependant, ces différentes études ont évalué séparément les deux aspects - d'un côté le dépistage et de l'autre le traitement. Or cette dichotomie peut conduire à des réponses incomplètes, voir même à des biais en termes de décisions considérées comme optimales. $\varepsilon$ n pratique, ne pas prendre en compte le devenir du patient dépisté, qu'il soit traité ou non, et les conséquences sur sa prise en charge future pour la société, témoigne davantage d'un objectif consistant à répondre à la question du « comment dépister? » et non pas à la question plus ambitieuse qui serait la question du bien-fondé du dépistage.

Aussi pour pouvoir se prononcer sur le bien-fondé d'allouer des ressources à un dépistage du VHC, et si c'est le cas, de pouvoir déterminer les populations qui pourraient être concernées par un dépistage systématique du VHC, le décideur doit pouvoir comparer des programmes de santé entre eux sur la base d'indicateurs de résultats ne se limitant pas au nombre de cas dépistés. Ainsi, à la suite de l'étude réalisée en 1997 [8], nous nous sommes efforcés dans une deuxième étude de prendre en compte, au moins en partie, les bénéfices escomptés du dépistage en raisonnant cette fois en nombre de pathologies évitées à la suite d'un dépistage et d'un traitement précoces [27]. Les résultats montraient que le dépistage des populations à risque était producteur à long terme d'économies par rapport à l'absence de dépistage. Et les ordres de grandeur des rapports coût-efficacité étaient cette fois compris entre 18430 et $99150 €(121000$ et $651000 \mathrm{~F})$ pour la stratégie ELISA, respectivement chez les usagers de drogues et dans la population générale. La stratégie de dépistage la plus efficace en fonction du coût était la stratégie comprenant un premier ELISA, suivi d'un deuxième ELISA en cas de résultat positif.

Cependant, notre étude, comme la plupart des études qui cherchent à exprimer un résultat sous la forme d'un coût par année de vie gagnée se heurte à un ensemble de limites : une connaissance imprécise de l'histoire naturelle de la maladie, de l'efficacité à long terme des 
bi- et tri- thérapies, du vécu de l'infection à VHC par le patient, ou encore de l'observance du traitement. Dans les populations à haut risque d'infection pour le VHC, particulièrement chez les usagers de drogue (prévalence $=80 \%$ ), les bénéfices devraient clairement prendre en compte les conséquences du dépistage sur les comportements à risque de contamination, et par conséquent sur le nombre de contaminations évitées grâce à un dépistage du VHC dans cette population. Les limites que rencontre l'analyse de décision sur l'infection par le VHC pourraient être en partie levées au regard des connaissances acquises sur l'infection par le VIH. Et ce pour deux raisons : d'une part, la découverte du virus de l'immunodéficience humaine est plus ancienne, et les études ont été nombreuses, que ce soit sur le plan économique, sociologique ou psychologique. Certains outils, comme certaines données pourraient effectivement s'appliquer au VHC, en attendant d'avoir suffisamment de recul sur la maladie. Par exemple, on pourrait évaluer l'observance des traitements VHC à partir des travaux déjà réalisés sur l'infection à VIH [28, 29], ou encore approcher les modélisations de l'évolution de l'hépatite $C$ en s'appuyant sur les modèles élaborés pour le VIH [30,31]. D'autre part, étant donnée la prévalence importante de l'infection à VHC chez les patients infectés par le VIH $(40 \%)$, il importe que l'analyste prenne en compte la fréquence de la coinfection et ses conséquences sur l'histoire naturelle de l'hépatite $C$ et sur sa prise en charge comme un élément déterminant dans l'évaluation économique du dépistage du VHC chez les usagers de drogues. Les nombreuses interactions entre le VIH et le VHC, même si elles ne sont pas encore bien comprises, nécessitent d'être considérées comme un facteur déterminant dans l'évaluation médico-économique du dépistage et de la prise en charge thérapeutique de l'infection à VHC. On peut espérer en effet que la prise en compte de la coinfection dans l'évaluation économique de l'infection à VHC aide, entre autres, à la diffusion de nouveaux schémas thérapeutiques, et surtout à une mise en œuvre de politiques de dépistage et de prise en charge plus adaptées, notamment chez les usagers de drogues.

\section{Conclusions}

Les décideurs dans le domaine de la santé semblent avoir pris conscience de la nécessité de contrôler l'offre de soins et d'arbitrer par conséquent entre efficacité, rentabilité et équité. II faut souligner que, dans ce domaine, décider de ne rien faire ou poursuivre une stratégie sans en analyser les conséquences économiques correspond toujours, au bout du compte, à une décision ayant néan- moins un impact économique, médical et social. II devient donc indispensable que se développent des méthodes formalisées d'évaluation permettant d'apprécier si ce qui est fait est réellement efficace et utile au regard des ressources utilisées pour y parvenir. C'est ce que se propose d'effectuer l'évaluation économique.

Par ailleurs, on peut espérer qu'un certain nombre d'incertitudes qui affectent la précision et la robustesse des estimations de l'ensemble des études économiques évoquées ci-dessus pourront être mieux documentées à l'avenir. En tout état de cause, les travaux déjà publiés, en rendant plus transparents les rendements décroissants de différentes stratégies alternatives de dépistage et de traitement, permettent d'éclairer les débats sur les seuils de ressources au-delà desquels la collectivité doit considérer que les ressources ne sont plus dépensées de façon socialement utile et produiraient plus de bien-être (c'est-à-dire, par exemple, sauveraient un plus grand nombre de vies humaines ou satisferaient mieux d'autres besoins) si elles étaient affectées ailleurs. On doit donc s'interroger sur le « coût d'opportunité » des ressources qui seront ainsi dépensées, et notamment sur leurs usages alternatifs possibles. A titre d'exemples, les évaluations économiques sur la prise en charge thérapeutique du VHC suggèrent que les traitements, y compris les plus coûteux et performants, aboutissent à sauver des années de vie pour des ratios jamais supérieurs à $15 \quad 000 / 23000 €(100$ 000/150 000 F) par année de vie gagnée. En revanche, I'analyse socio-économique évaluant le rapport coûtefficacité de l'introduction de la PCR dans le dépistage systématique des dons de sang pour le virus de l'hépatite C [14], montre que cela reviendrait à accepter de consacrer des coûts de l'ordre de plusieurs millions d'€ pour gagner potentiellement une année de vie «statistique » supplémentaire dans la population des transfusés. Fort de cette comparaison, le coût global additionnel de l'introduction de la PCR sur des pools de sang, s'il était attribué à la prise en charge de patients infectés par le VHC permettrait de traiter par interféron et ribavirine plus de 1100 personnes.

Cependant, si l'évaluation économique est un outil qui peut aider à la prise de décision, il n'en existe pas moins, parfois, des contradictions entre les résultats de l'évaluation économique et le choix du décideur qui en résulte. Ce qui nous amène à penser que le poids que peuvent représenter les résultats de l'évaluation économique dans la balance décisionnelle est toutefois affaibli par des considérations d'ordre politique, le critère coût-efficacité étant relégué à une fonction plutôt informative que décisionnelle. $\diamond$ 


\section{SUMMARY}

Appraisal of economic evaluations

of treatment and screening for hepatitis $C$

The interest of economic approaches in health is the possibility of organising medical programs likely to be carried out, on the basis of two criteria (medical effectiveness and costs), and of helping to set objectives of public health, in the short or the long term, compatible with the resources available. This article presents a review of specific literature of economic evaluations which have contributed to the definition of optimal policies as regards to screening and treatment of hepatitis $C$. The results of these economic evaluations show, on the one hand, that in spite of a high cost, the screening of populations at risk for HCV infection is generator of long-term economy compared to the absence of screening, and suggest in addition, that treatments, including the more expensive and more efficient, result in saving life years with ratio never greater than those of health interventions actually undertaken in industrialised countries. The decision analysis applied to the screening and treatment of hepatitis $C$ shows an illustration of decreasing outputs: the more screening is extended to groups of population where the prevalence of infection is low and the more it will be necessary to use resources to identify an additional case. Thus, those economic evaluations enlighten the debates about the thresholds of resources beyond which resources are not spent any more in a socially useful way. $\diamond$

\section{RÉFÉRENCES}

1. Couturier $\varepsilon$, Brossard $Y$, Rotily M, et al. Séroprévalence des anticorps anti-VHC dans un échantillon exhaustif de femmes ayant terminé une grossesse en régions lle de France et PACA. BEH 1996; $5: 19-20$.

2. Dubois F, Desenclos J, Mariotte N, Goudeau A. Séroprévalence et facteurs de risque de l'hépatite $C$ dans un échantillon national de volontaires à un examen de santé de la Sécurité Sociale. BEH $1996 ; 5$ : 17-9.

3. Couroucé AM, Pillonel J. Estimation of risk of virus transmission in hepatitis B and $C$ and human retrovirus via transfusion of labile blood derivatives. Transf Clin Biol 1996 ; 3 : 858-62.

4. Deuffic S, Poynard T, Valleron AJ. Correlation between hepatitis $C$ virus prevalence and hepatocellular carcinoma mortality in Europe. J Viral Hepatitis $1999 ; 6$ : 411-3.

5. Drummond M, Stoddard G, Torrance G, O'Brien B. Méthodes d'évaluation économique des programmes de santé. Paris : Economica, 1998.

6. Vergnon P, Colin C, Jullien A, et al. Evaluation médicoéconomique du dépistage des hépatites $C$ et non- $A$ non- $B$ non- $C$ sur les dons du sang. Rev Epidemiol Sante Publ 1996; 44 : 66-79

7. Sailly J, Lebrun T, Coudeville L. Approche coût-efficacité du dépistage des virus VIH VHB VHC HTLV chez les donneurs de sang en France. Rev Epidemiol Sante Publ 1997 ; 45 : 131-41.

8. Rotily $M$, Loubière $S$, Nixon J, Bourlière $M$, Halfon $P$, Moatti JP. Analyse socio-

GLOSSAIRE

Coût moyen

On appelle coût moyen (CM), le coût total de la stratégie divisé par l'efficacité produite par cette même stratégie.

Coût marginal

On appelle coût marginal $(\mathrm{Cm})$, le supplément de coût résultant de la production d'une unité supplémentaire d'efficacité. La notion de coût marginal intervient lorsque l'on compare deux stratégies entre elles.

\section{Rendements décroissants}

Plus on étend une stratégie médicale à un groupe plus large d'individus, et plus il faut de ressources pour obtenir une unité d'efficacité supplémentaire.

PALY

Cet indicateur pondère l'efficacité médicale (par exemple le nombre d'années de vie gagnées) par une appréciation de la qualité de la vie. Cette approche consiste à ajuster la durée de vie affectée par le résultat de santé à l'aide de valeurs exprimant la qualité de vie dans les états de santé (la valeur attribuée aux différents états de santé étant le plus souvent exprimée par une échelle allant de 0 : mort à 1 : santé parfaite). Pour obtenir l'indicateur QALY, on multiplie le coefficient de pondération de la qualité de vie associé à un état de santé par la durée de temps passé dans cet état.

économique de différentes stratégies de dépistage de I'hépatite chronique C dans la population française. Gastroenterol Clin Biol 1997 ; 20 : S33-40.

9. Laplane K, Jakiche A, Sugano D, Weng C, Carey W. Hepatitis C infection risk analysis: who should be screened? Comparison of multiple screening strategies based on the National hepatitis surveillance group. Am J Gastroenterol 1998 ; 93 : 591-6.

10. Kaur S, Rybicki L, Bacon B, et al. Performance characteristics and results of a large-scale screening program for viral hepatitis and risk factors associated with exposure to viral hepatitis B and C: results of the national hepatitis screening survey. Hepatology 1996 ; 24 : 979-86.
11. Schaffer P, Allemand $H$. Evaluation économique du dépistage du cancer du col de l'utérus. Concours Medical 1995 ; 32 : 2501-14.

12. Andrew W, Whynes D. Filtering strategies in mass population screening for colorectal cancer. Med Dec Making $1992 ; 2$ : 2-7.

13. Moatti JP. L'évaluation économique : un complément nécessaire de l'évaluation médicale. In: L'évaluation médicale: du concept à la pratique. Paris : Flammarion-MédecineSciences, 1994 : 71-4.

14. Loubière $S$, Rotily $M$, Durand-Zaleski I, Costagliola D, Moatti JP. L'introduction de la PCR dans le dépistage du virus de l'hépatite $C$ dans les dons de sang: du mésusage du principe de précaution. Med Sci 2001 ; 17 : 344-9. 
15. Hillner B, Smith T, Desh C. Assessing the costeffectiveness of adjuvant therapies in breast cancer using decision analysis model. Breast Cancer Res Treat $1993 ; 25: 97-105$.

16. Moonrey G, Hall J, Donaldson C, Gerard K. Utilisation as a measure of equity: weighing heat? J Health Econ 1991 ; $10: 475-80$.

17. Moatti JP. Ethical issues in the economic assessment of health care technologies. Health Care Analysis 1999 ; 7 : 153-65.

18. Micks RA, Cullen JW, Jackson MA, Burry F. Hepatitis B virus vaccine. Cost-benefit analysis of its use in children's hospital. Clin Pediatr 1989; 28 : 359-65.

19. Joliot $\varepsilon$, Vanlemmens $C$, Kerleau D, Le Gales C. Analyse coût-efficacité du traitement de l'hépatite chronique C. Gastroenterol Clin Biol 1996 ; 20 : 958-67.

20. Michel P, Merle V, Gourier C, Hauchain $\mathrm{P}$, Colin R, Czernichow P. Efficience comparée de trois stratégies de prise en charge de I'hépatite chronique C : influence sur le risque de cirrhose à 8 ans.

Gastroenterol Clin Biol 1996 ; 20 : 47-54.

21. Bennett W, Inoue $Y$, Beck R, Wong J, Pauker S, Davis G. Estimates of the costeffectiveness of a single course of interferon alpha $2 b$ in patients with histologically mild chronic hepatitis C. Ann Intern Med 1997 ; 127 : 855-65.

22. Shiell A, Briggs A, Farrell G. The cost-effectiveness of alpha interferon in the treatment of chronic active hepatitis C. Med J Austr $1994 ; 160: 268-72$.

23. Dusheiko G, Roberts J. Treatment of chronic type $B$ and $C$ hepatitis with interferon alfa: An economic appraisal. Hepatology 1995 22 : 1863-73.

24. Kim W, Poterucha J, Gross J, Dickson $\varepsilon$, Evans W. Costeffectiveness of 6 and 12months of interferon-alpha treatment for chronic hepatitis C. Ann Intern Med 1997 ; 127 : 866-73.

25. Wong J, Bennett W, Koff R, Pauker S. Pretreatment evaluation of chronic hepatitis C: risks, benefits and costs. JAMA $1998 ; 280$ : 2088-93.

26. Wong JB, Poynard T, Ling MH, Albrecht JK, Pauker SG. Costeffectiveness of 24 or 48 weeks of interferon alpha- $2 b$ alone or with ribavirin as initial treatment of chronic hepatitis C. International Hepatitis Interventional Therapy Group. Am Gastroenterol 2000 ; 95 : 1524-30.

27. Loubière $S$, Rotily $M$, Portal I, Bourlière M, Moatti JP. Évaluation économique des stratégies de dépistage de I'hépatite chronique C. Med Mal Infect 1999 ; 29 :337-44.

28. Escaffre N, Morin M, Bouhnik $A D$, et al. Intravenous drug users' adherence to HIV antiretroviral treatments: physicians' beliefs. AIDS Care $2000 ; 12: 723-30$.
29. Moatti JP, Carrieri MP, Spire $B$, et al. Adherence to Haart in French HIV-infected injecting drug users: the contribution of Buprenorphine drug maintenance treatment. The Manif 2000 study group. AIDS 2000 ; 14 : 151-5.

30. Longini IM, Clark S. Statistical analysis of the stages of HIV infection using a markov model. Stat Med 1989; 8 : 831-43.

31. Chancellor JV, Hill AM, Sabin CA, Simpson KN, Youle M. Modelling the cost effectiveness of Lamivudine/Zidovudine combination therapy in HIV infection. Pharmacoeconomics 1997 ; 12 : 54-66.

\section{TIRÉS À PART}

S. Loubière 